Hill we see that the question is not one of municipal, county or state, but really an international problem, because Dr. Hill wants to remove from the United States the stigma of being the fourteenth on the list in regard to the mortality of the mother and bring us up to the first place. The necessary information and influence to inspire our government to this action must be found in the 144,000 or more members of the medical profession, many of whom are members of this Association. No work is more important. It is just as important to conserve the life of the mother as that of the newborn child. The Children's Bureau of Philadelphia is seeking to save at least 100,000 of the 300,000 children who die before the sixth year.

Dr. J. H. Carstens, Detroit: We can accomplish this work in various ways. We now teach better obstetrics than we have in the past. We are requiring that students actually attend cases of confinement, and they are learning the details of the management of the work. In the college with which I am connected we require the student to attend twelve cases and to keep an accurate record of the details. The midwife does not seem to have a large number of infant mortalities. This, however, is only apparent, and it must be remembered that in a serious case the midwife calls in a physician. $\mathrm{He}$ has to sign the death certificate and gets the blame. It is a matter of education of the public. We must agitate the question until boards of health and nurses educate the people so that every woman may know that when she is pregnant (although the process is a physiologic one), it may become a very serious surgical case and that she should consult a doctor. Of course, to educate the people necessitates the help of boards of health, nurses and investigators of all kinds. The suggestion made by Dr. Hill of having the United States take hold of the matter seems eminently proper. If the United States can afford many thousands of dollars to take care of hogs it certainly can afford hundreds of thousands to take care of women and children. We should use our influence with our congressmen. If we do this we can probably get Congress to do something for the health of the pregnant women. Much could be done, as Dr. Hill has said, with a health officer a member of the cabinet. From him this work would radiate throughout the country and we should have in every district a health officer instructing the people in the matter of health.

Dr. Irving F. Stein, Chicago: In Chicago we are doing much the same work as that described by Dr. Hill. At the Michael Reese Hospital we have a prenatal clinic in which we have seen as many as seventy-eight women in one morning. The clinic meets twice a week. The blood pressure and urinalysis is taken at every visit. The first visit comprises a complete physical examination, including observations of the thyroid, the teeth, the breasts, the heart, the limbs for varices, etc. We try to educate the women in the care of their teeth and in the wearing of abdominal supports. If they wear corsets, we show them how to wear them properly. The incidence of eclampsia has been markedly reduced since the inauguration of the clinic. The pathologic cases are recognized early, are sent into the maternity for observation and consultation, and cared for before they become "neglected cases."

Dr. IRA L. Hill, New York: In my reference to the midwives I brought the matter up not to praise them but to show how much their work had progressed since they were under supervision. Regarding the investigations being made in Washington by the Department of Labor through the Children's Bureau, it is interesting to know that the greatest inspiration for any one who has had occasion to do any work in the study of prenatal statistics comes from that bureau, and that the two hundred and some odd thousand dollars which they have had for this work represents a tax of a third of a cent a year for all the inhabitants. Our government, therefore, could probably afford to spend considerably more and still not be working any injustice to the people.

Experimentation.-It is too often overlooked that for century after century Nature has been pitilessly performing her crude and cruel experiments and killing millions of human beings every year.-W. W. Keen.

\section{A NEW PRINCIPLE IN THE SURGI- CAL TREATMENT OF BRAIN TUMORS *}

A. C. STRACHAUER, M.D.

Associate Professor of Surgery, University of Minnesota MINNEAPOLIS

Craniotomy for brain tumor, with or without focal symptoms, not infrequently fails to disclose the neoplasm. The evidence of increased intracranial pressure will be manifest by various degrees of brain bulging, by a flattening out of the convolutions, by obliteration of the sulci and by diminished or absent brain pulsation. Inspection, palpation, and exploration by incision or aspirating needle all fail to locate or reveal the cause. The tumor may be so deep within the brain substances or at such a stage of development as to be inaccessible. The lesion may be an infiltrating glioma, which cannot be macroscopically differentiated from the normal brain, or the craniotomy may not have been formed at the proper site. The wound is closed, temporary benefit is conferred by the decompression, and in time the patient dies.

Definite localizing information may develop after decompression in cases in which focal symptoms have been absent. In these cases, patients have been not infrequently reoperated on and the brain tumors successfully enucleated. The cases with focal symptoms before operation, and those without, which do not develop localizing data after negative exploration, are considered hopeless and the patients are permitted to die. The necropsy shows the tumor, or no further investigation is made. My discussion is limited to this latter type, the "hopeless" case, which deserves further consideration and should not be given up, for the tumor may and can be removed at times, as illustrated in the following case from the University Hospital.

\section{REPORT OF CASE}

CASE 11487.--E. L. B., a man, aged 29 years, was admitted to the neurologic service of Dr. A. S. Hamilton, July 8, 1917, on the application diagnosis of cerebral syphilis, complaining of excruciating headache, impaired vision, spastic paralysis of the left arm and leg, and general weakness. He had had several attacks of unconsciousness lasting from one to two hours. There was slight incontinence of the bowel and the bladder. The administration of potassium iodid and mercury had been followed by improvement of symptoms. The blood and spinal fluid Wassermann reactions were negative. Bilateral papilledema were present, more pronounced in the right eye ground. He had several typical jacksonian seizures while in the neurologic service, the attacks beginning in the left foot and extending up the body, involving the leg, the arm and the face. The diagnosis of cerebral neoplasm in the right rolandic area was made, and the case was transferred to the surgical service.

July 24, 1917, a large osteoplastic flap opening was made over the right motor area of the brain. When the dura was opened the brain began to bulge. The intracranial pressure was extreme, the cortical vessels were engorged, and the surface of the brain became smooth and flat, the sulci and convolutions being flattened out and obliterated. Inspection, palpation and exploration with Cushing's aspiration needle failed to disclose the neoplasm. Aspiration of the lateral ventricle was performed. The cerebral pressure was so great that the bone trap door had to be removed to effect a closure. The case was considered as being a deep-seated lesion or an

* Read before the Section on Surgery, General and Abdominal, at the Sixty-Ninth Annual Session of the American Medical Association, Chicago, June, 1918 
infiltrating and irremovable glioma. In other words, the case was in the "hopeless class" described before.

The patient was definitely improved by the decompression and asserted that his unbearable headache had gone the same day of the operation. The acuity of vision improved, as did the choked disks. The wound healed per primum. Bulging of the brain under the scalp was present.

There gradually developed an accumulation of cerebrospinal fiuid and a cystoid degeneration of the scar forming a cystic mass the size of a fist. This mass transmitted light and sunshine like a hydrocele, was soft, fluctuating and painless. Aspiration of the fluid was considered. Four months had now elapsed since the exploratory operation.

In studying the case I began to develop the idea that even though it had been impossible to locate and to remove the tumor at the previous operation, change and growth might have taken place in the interim, such as to make the neoplasm now accessible. A deeply seated growth might have come to the surface, or nearer to the surface, and now be removable.

Nov. 16, 1917, the patient was reoperated on. An incision was made through the old scar, the fluid was evacuated and the flap elevated. When the brain was uncovered, a thin, blue-walled cyst of the size of a goose egg was disclosed. Under the most gentle manipulation the cyst ruptured. On further examination the cyst was found to be embedded in a definite tumor mass and the lesion was evidently one of cystic development in a glioma or sarcoma, the latter neoplasms being prone to cystic degeneration. The overlying cortex was incised to facilitate removal. During this incision the anesthetist remarked that the left side of the face twitched. There was a definite line of demarcation between the tumor and the surrounding brain. By the most careful manipulation with the index finger, working to a depth of its length, and with the assistance of a teaspoon, the tumor, the size of an orange, was freed and delivered. The remaining cavity was gently packed with a hot cotton wad to control oozing, and several cerebral vessels were ligated. On removal of the hemostatic pack the brain gradually filled in the cavity and at the same time of closure the cavity had become practically obliterated. The patient made an uneventful recovery.

On the second day the movements of the arm were definitely improved over the preoperative condition. The mentality improved and in several weeks the patient was up and walking with perfect control of his sphincters.

\section{IMPORTANCE OF THE CASE}

A deep-seated tumor had evidently developed, and by the assistance of the cystic degeneration had come to the surface during the interval between the two operations. The decompression may have had a favorable influence in causing the growth to point and to be in a degree spontaneously extruded.

This case establishes a precedent, and illustrates a principle which, if followed, will in some cases prolong and in others save lives from the class which have been given a chance and then considered "hopeless." It turns defeat into victory. The practice in the foregoing hopeless cases should be reoperation at a favorable time, which will vary in different cases. The patients should not be given up. Without reoperation, the patient has been given only half a chance.

The medical profession as a whole is too pessimistic in its attitude toward neurologic surgery. Practically every field of successful modern surgery has had to pass through its trying and discouraging period of unsatisfactory results. This has been particularly true of the brain. Neurologic surgery is emerging from its dark age and is becoming a comparatively safe practice in the hands of the specially trained.

When we consider that the inevitable fate of the brain tumor patient is death, which becomes a kind relief from indescribable physical and mental suffering including blindness, the single successful removal of such a growth with complete restoration to the normal is a reward and satisfaction that cannot be measured.

I have performed thirty-six craniotomies with two operative mortalities. The first was a tumor of the cerebellum, the patient being somnolent and so far moribund that he died twenty-four hours after an uncomplicated suboccipital decompression. The second died from a large, deeply seated, irremovable cerebral tumor. Bilateral decompression was performed but was insufficient to relieve the medullary compression. There were two cases of postoperative pneumonia, which would ordinarily be considered "post ether" and attributed incorrectly to the anesthetic, had not the one followed a local anesthesia decompression.

\section{ADVANTAGES OF DECOMPRESSTON}

The following views and principles of practice are based on the work of a number of neurologic surgeons and on the writer's personal experience. livery patient with a brain tumor is entitled to a chance and at least to a decompression. Any degree of papilledema calls for an immediate decompression, the operation being imperative and emergent. Organization obtains very early and when optic atrophy has once begun, it may be impossible even to halt its progress. Decompression after atrophy is "locking the barn after the horse is gone." With choked disks present, decompression may be just as imperative to save sight with a positive Wassermann reaction as with a negative. The decompression should be large enough actually to relieve the intracranial pressure, otherwise its performance is worse than useless. It should measure 5 by $7 \mathrm{~cm}$. at least. I perform the Cushing subtemporal musclesplitting operation for cerebral growths and suboccipital decompression for tumors below the tentorium. The subtemporal operation may be performed under local anesthesia when indicated. The dura is always opened, preferably by flap formation, a marginal cuff being left to cover the decompression circumference.

Decompression alone brings great relief for the insufferable headache, saves the vision and at times even in case of irremovable growths, restores the patient for a long period of usefulness. It is nearly unbelievable, after decompression or large osteoplastic flap operation, to have patients say, immediately on coming out from under the anesthetic, or on the day following, that their excruciating headache is gone and that they are comfortable.

A preliminary subtemporal decompression is performed in nearly all cerebral growths on the side opposite the suspected lesion. This makes the ultimate decompression in irremovable growths bilateral and symmetrical. The onesided bulging at times causes undue tension on the brain stem and interference with brain function due to the distortion. The relief afforded from intracranial pressure makes the second operation for removal safe and less hemorrhagic, and permits a more frequent return of the osteoplastic trap door. Aspiration of the ventricles frequently relieves the pressure and is a routine procedure in extreme pressure.

The problem is that of dealing with a brain under such pressure that the vital centers in the medulla are on the verge of paralysis with the death of the patient as a consequence. Any additional compression on the already overcrowded medullary centers as caused by the passage of the Gigli saw between the trephine openings may be just sufficient to be the "last straw" and cause death. The preliminary decompression guards against this occurrence. 
From a week to ten days after the preliminary decompression, an osteoplastic trap-door opening is made over the diagnosticated site of the tumor. A large, generous opening is of the greatest importance, so that, no matter what condition may be found, it may be adequately dealt with. Lesions have been missed by being just outside a small exposed area of the brain. Patients do not die from having large osteoplastic flaps, properly formed, but they do from small ones. The intracranial pressure previously described is relieved by a large exposure. On the introduction of the finger and instruments into the brain substance for the removal of the tumor, room is provided for the expansion and bulging of the brain and the overcrowded vital, medullary centers are again saved from paralysis. In other words, fatal medullary compression is avoided. This, in my opinion, is one of the most important factors in the successful removal of brain tumors, yet it is not adequately appreciated.

During the anesthetization of the patient in brain tumor operations, cessation of respiration is of rather common occurrence, being due to the increased pressure on the already crowded medullary respiratory centers by the congestion of the brain caused by the ether. Clinical observation and animal experimentation have established the fact that respiration stops under cerebral compression long before cessation of heartbeat, and that the cardiac function can be kept up for long periods by artificial respiration.

\section{MITIGATION OF SHOCK}

Another factor responsible for death in brain operations is shock. Shock in general surgery is in great measure synonymous with hemorrhage. The excessive loss of blood indirectly produces shock. Hemorrhage is under the control of the operator and the greatest attention to hemostasis is essential to success. Horsley's bone wax and the wooden peg are employed for osseous hemorrhage and Cushing's cotton compresses and the ligature for cerebral.

In my experience, the Haidenhein hemostatic suture has proved more satisfactory than the Cushing tourniquet or Makka's clips for the control of bleeding from the scalp.

Injudicious handling of the brain and the infliction of compression and concussion directly produce shock. The greatest care and gentleness is to be exercised. Rapidity of operating is to be avoided. I believe that direct shock of the latter type is as much subject to the law of dosage and concentration as is strychnin and like toxic drugs. A weak solution administered over a period of time will be tolerated, while a concentrated dose will kill. Too speedy operating in neurologic surgery is equivalent to the inflicting of a concentrated dose of shock. This is in direct contradiction to the regular statement of textbooks which all laud rapid removal of the tumor. This $I$ believe to be absolutely incorrect.

As illustrative of the technic of diminishing shock, in removing a glioma the size of a goose egg from the upper right motor region in a patient at the Abbott Hospital, after incising the cortex, I consumed about ten minutes or more in practically watching and gently assisting the spontaneous extrusion of the neoplasm. The case resembled an unassisted obstetric delivery. Forceps might have been applied, but were not. Here likewise the tumor was spontaneously delivered by the intracerebral pressure.
The osteoplastic trap door is cut with a broad bevel so that a solid replacement may be effected and firm sutures and snug bandaging be free from exerting pressure on the underlying brain. In irremovable brain tumors, it is desirable to rongeur away a large portion of the base of the hinge area of the bone flap. This provides for generous decompression, and by the return of the beveled margin, the undisturbed muscles over the decompressed area control herniation of the brain.

I believe that technic should be simple and that the trephine, Gigli saw, and bone-biting forceps, which always work, inflict less insult and are better and safer than electrically driven circular saws, drills and burs, which are supposed to lock when through the skull, and which in reality do not always do so. As a hand-sewed shoe is superior to a machine-made one, so is a hand performed operation superior to one performed by machine.

\section{CONCLUSIONS}

Brain tumor cases following negative craniotomy are not necessarily hopeless. A deep-seated, inaccessible lesion may develop in time, come to or near the surface, and so become removable. The decompression may have a favorable influence in causing the growth to point, and to be in a degree spontaneously extruded. Such a case given a single exploration has had only half a chance. Reoperation may turn defeat into victory.

Shock is the principal cause of operative deaths in neurologic surgery: Direct shock is subject to the laws of concentration and dosage. Rapid operating is equivalent to the infliction of a concentrated dose of shock and is to be avoided.

Indirect shock is synonymous with hemorrhage and is within the control of the operator.

Large decompressions and operative field exposures relieve and avoid medullary compression and are essential to success.

In the hands of the specially trained and experienced the operative mortality in neurologic surgery compares favorably with that in other branches of surgery. Without surgical intervention, the inevitable fate of the patient afficted with a brain tumor is death. Surgery alone can save sight, alleviate indescribable physical and mental suffering, prolong life and at times establish a cure.

\section{ABSTRACT OF DISCUSSION}

Dr. L. L. McArthur, Chicago: This has been a recognized procedure for many years. Ten years ago I saw Victor Horsley remove for the second time at the third operation, a tumor of the brain which had recurred in loco, by repeating the elevation of the flap and by simple enucleation with the finger. At the first operation the tumor was not localizable. but it presented at the second operation. Localization of brain tumors is the great difficulty. He demonstrated how easy it was to be mistaken as to the location of brain tumors on the basis of symptoms. By putting a needle into the dentate nucleus of the cerebellum of a monkey he could get a lateral deviation of both eyes to the right, which symptom has been taken to indicate a cerebellar tumor. He put the same needle an inch ligher up in the posterior occipital lobe and demonstrated that the same phenomenon obtained. He moved it forward to the motor area, and there again over a third place, widely removed, he produced a typical deviation of the eyes to the right so that the difficulty of localization is still a bugbear. The statement the author makes that the localization sometimes becomes evident after a decompression is an important one. Its importance is 
emphasized more promptly by the more simple procedure of making a callosal leak in the roof of the lateral ventricle on the side on which the neurologists believe the tumor to be. By doing so the roof of this ventricle leaks. It leaks outward instead of inward, and relief of the tension and decompression is obtained with the least possible mutilation. Then the focal symptoms may develop which will enable the surgeons to make the operative procedure, which is recommended by the author. The patient should have, then, the opportunity for the tumor to be crowded by the intracranial pressure toward the lines of least resistance, and should again be explored when the time has sufficiently elapsed to enable him to do so. Not infrequently it is possible in these frightfully deformed cases which follow these lateral osteoplastic flaps to remove a tumor which had deformed the patient until he is an object of repugnance, by making just such an exploration. The procedure and the recommendations of the author are to be recommended.

Dr. Artuur C. Strachader, Minneapolis: It is the regular practice, or so frequently so, after a negative exploration, to permit these patients to die. List winter I saw a case in which a negative subtemporal decompression had been performed. The patient died with a large cyst, which undoubtedly would have been amenable to treatment. There is nothing new about reoperating in cases where nothing was found. then have localizing symptoms develop, operate and remove the tumor. That has been a recognized practice for many years. It is common to operate these cases a second, third and even a fourth time. But it is the cases in which nothing has been found by the evidence of intracerebral pressure, that I particularly wanted to call attention to, and in which following decompression no further symptoms of localizing value developed. These patients should not be permitted to die, because there is a possibility of removing a neoplasm.

\section{UTERINE INERTIA}

\section{SUMMARY OF A SERIES OF CASES *}

PAUL TITUS, M.D. PITTSBURGH

The classification and analysis of cases of uterine inertia are somewhat difficult because the term uterine inertia is indicative of a more or less intangible condition. Inertia of a laboring uterus cannot be established on a time basis, since there are a great many factors which make it a relative rather than an absolute entity. For instance, a primipara delivering herself of an average sized infant, and a multipara giving birth to a small baby after her uterine muscle tone - has been impaired by a number of pregnancies closely following each other, might both consume the sane length of time in accomplishing a different amount of work. This normal period of time for the first woman to be in labor would be abnormal for the second woman, and it could be said of the latter that she had inertia because her pains were of poor quality in regard to length and strength.

It is not known exactly why a woman falls into labor at the end of about 280 days of pregnancy. It might just as well have been normal, so far as the welfare of the fetus is concerned, for a preguancy to consume eleven or twelve lunar months instead of ten. As it is, we can only say that when the fetus is mature, namely, at the end of ten lunar months, labor usually begins. If the stimulus to its induction is normal and the uterine muscle tone is good, labor will go on actively and progressively. On the other hand, some cases in which labor has been artificially induced by

* Read before the Section on Obstetrics, Gynecology and Abdominal Surgery at the Sixty. Ninth Annual Session of the American Medical Surgery at the Sixty. Ninth Annual
Association, Chicago, June, 1918. means of bags and bougies, as well as many in which labor is instituted by premature rupture of the membranes, exhibit uterine inertia of varying degrees.

\section{PRIMARY AND SECONDARY INERTIA}

The propriety of classifying uterine inertia as primary or secondary is extremely doubtful. In fact, true uterine inertia is practically always primary, since it is due to some inherent fault in uterine muscle tone, or at least to a lack of normal stimulus to its contractile power. In making the distinction between so-called primary and secondary inertia, it should be pointed out that the term inertia is defined as "an incapability of matter to change its state whether that be one of rest or motion," or a "sluggishness," or "an indisposition to exertion." This may be secondary to some constitutional debility of the patient such as pulmonary tuberculosis, or to an early rupture of the membranes, or over distention of the uterus from either hydramnios, twins, or frequent pregnancies, and in this respect the term secondary might be permissible. That is not, however, the significance given it by common usage. Inertia, arising early in labor from causes like these, is commonly called primary, while the term secondary inertia has come to convey the idea of a sluggishness arising later in the course of a labor, in which the pains had been normal up to that point. We are told, for instance, that neglected labor in contracted pelvis or with malpositions develops into secondary inertia, whereas I maintain that this is not uterine inertia, but rather uterine exhaustion.

It will be said that this is splitting hairs, and that, if the uterine contractions are ineffective, because the uterus is inert or exhausted, as the case may be, the conditions which obtain are identical and the general treatment is similar in both cases. This is not quite correct, however, because the distinction between inertia and exhaustion has a definite bearing on both the treatment and the prognosis in the two instances.

\section{DISTINCTION BETWEEN INERTIA AND EXHAUSTION}

The origin of the two conditions is not identical, for inertia is due to an inherent fault while exhaustion follows a long continued effort against an obstacle. Prophylactic treatment is obviously different. In the true inertia there is comparatively little to be done by way of prevention, whereas one should anticipate or do away with such obstacles to labor as contracted pelvis or occiput posterior and face presentations, to mention a few examples. In this series I have purposely avoided discussing cases of uterine exhaustion since this is a question for treatment directed at the underlying cause of the exhaustion.

True inertia is first noticed in the first stage of labor. With unruptured membranes a prolongation of the first stage is not often serious and usually the patient may be carried on indefinitely by such means as narcotics, or the mild stimulation of hot enemas, strychnin, quinin, etc., according to the needs of the case.

Individuals who are below par physically, whether from wrong living or some debilitating illness or some actual physical defect, are liable to be nervous and intolerant when brought face to face with any stress or strain, and the same condition which is a cause of this may readily be a factor in the occurrence of uterine inertia. Such a person stands the nagging and delay of a sluggish uterus very badly, and will often have exceed- 